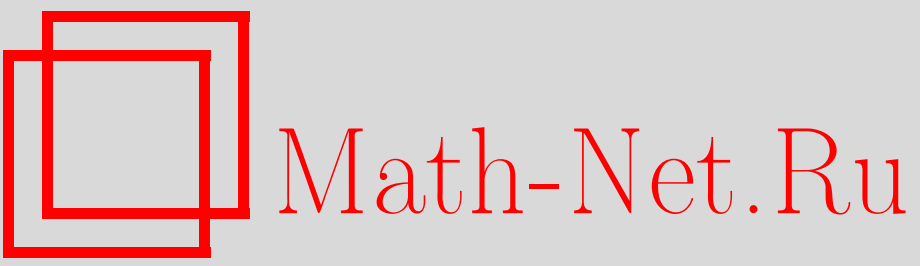

D. K. Durdiev, Zh. Z. Nuriddinov, On investigation of the inverse problem for a parabolic integro-differential equation with a variable coefficient of thermal conductivity, Vestn. Udmurtsk. Univ. Mat. Mekh. Komp. Nauki, 2020, Volume 30, Issue $4,572-584$

DOI: https://doi.org/10.35634/vm200403

Use of the all-Russian mathematical portal Math-Net.Ru implies that you have read and agreed to these terms of use

http: //www. mathnet.ru/eng/agreement

Download details:

IP : 54.197.217.227

April 26, 2023, 14:23:59 
MSC2010: 35R30, 35K70, 35M12

\author{
(C) D. K. Durdiev, J.Z. Nuriddinov
}

\title{
ON INVESTIGATION OF THE INVERSE PROBLEM FOR A PARABOLIC INTEGRO-DIFFERENTIAL EQUATION WITH A VARIABLE COEFFICIENT OF THERMAL CONDUCTIVITY
}

The inverse problem of determining a multidimensional kernel of an integral term depending on a time variable $t$ and $(n-1)$-dimensional spatial variable $x^{\prime}=\left(x_{1}, \ldots, x_{n-1}\right)$ in the $n$-dimensional heat equation with a variable coefficient of thermal conductivity is investigated. The direct problem is the Cauchy problem for this equation. The integral term has the time convolution form of kernel and direct problem solution. As additional information for solving the inverse problem, the solution of the direct problem on the hyperplane $x_{n}=0$ is given. At the beginning, the properties of the solution to the direct problem are studied. For this, the problem is reduced to solving an integral equation of the second kind of Volterra-type and the method of successive approximations is applied to it. Further the stated inverse problem is reduced to two auxiliary problems, in the second one of them an unknown kernel is included in an additional condition outside integral. Then the auxiliary problems are replaced by an equivalent closed system of Volterra-type integral equations with respect to unknown functions. Applying the method of contraction mappings to this system in the Hölder class of functions, we prove the main result of the article, which is a local existence and uniqueness theorem of the inverse problem solution.

Keywords: integro-differential equation, inverse problem, kernel, contraction mapping principle.

DOI: $10.35634 / \mathrm{vm} 200403$

\section{Introduction}

The theory and applications of integro-differential equations with an integral term of convolution-type with respect to a time variable play an important role in the mathematical modeling of heat and mass transfer. In these processes the convolution kernel describes the relaxation times of the heat flux. In the general case, in integro-differential equations of such kind, the convolution kernel takes into account the memory affects as usual. The fundamental point, when dealing with memory effects, is that the kernel cannot be considered a known function, since there are no ways to measure it directly. What we do is to reconstruct this kernel by additional measurements of a physical field, taken on a suitable subset of the body. Thus, the inverse problem arises, the study of solvability (the theorem of existence and uniqueness) of which is of great interest in the theory of inverse problems.

The dynamical representation of heat transfer processes is modeled quite accurately by including the past history of one or more time and space variables through memory kernel [1-4]. This phenomenon is governed by parabolic integro-differential equations with a time dependent memory kernel when the medium is homogeneous and a time-space dependent memory kernel when the medium is heterogeneous.

In the last years, various approaches have been applied for identification of heat source terms, of unknown boundary conditions, of memory kernel or spatially varying coefficients. The determining of the convective coefficients using different kinds of temperature measurements by means of inverse heat conduction were investigated in [5-10] (see also references therein). Authors in these works discussed the issues of existence, uniqueness and stability estimates of solution. Here a numerical approach for solving such problems has also been applied. 
In papers [11-13] the inverse problems of finding the time-dependent heat source with nonlocal boundary and integral additional conditions were investigated. The existence, uniqueness and continuous dependence of the solution of the inverse problem upon the data were established. Also, numerical results were presented and discussed. In the work [14] the inverse boundary value problem of heat conduction with a piecewise constant coefficient was considered. This problem was investigated using the Fourier series in eigenfunctions for an equation with a discontinuous coefficient.

Among the works devoted to the kernel determination problems in heat conduction equations, one-dimensional problems are most encountered, that is the problems of memory kernel determining depending only on a time variable. For example, in $[1,2,15]$ (see also the references therein) these problems were investigated based on a fixed point argument and derived the local in time existence and uniqueness of inverse problems. Additionally, for problem of [15] a numerical experiment was conducted.

The results on multidimensional problems of determining the kernel in parabolic integrodifferential equations are very rare. In this direction we only note the works [16-19]. In [16] author deals with the problem of recovering a memory kernel $k(t, \eta)$, depending on time $t$ and on an angular variable $\eta$, in a parabolic integro-differential equation related to a toric domain involved in $\mathbb{R}^{2}$. In the works $[17,18]$ the problems of determining a kernel depending on a time variable $t$ and $(n-1)$-dimensional spatial variable $x^{\prime}=\left(x_{1}, \ldots, x_{n-1}\right)$ entering into in integral term of a $n$-dimensional heat equation were investigated. The local unique solvability result was obtained. In [19] the uniqueness theorem of recovering kernel depending on all variables $t$ and $x=\left(x_{1}, \ldots, x_{n}\right)$ was proved for special case of kernels.

In the present paper we study an inverse problem to determine the temporal and $(n-1)$-dimensional spatially varying memory kernel for a parabolic integro-differential equation with a variable coefficient of thermal conductivity.

Consider the problem of determining functions $u(x, t), k\left(x^{\prime}, t\right), x=\left(x_{1}, x_{2}, \ldots, x_{n-1}, x_{n}\right)=$ $=\left(x^{\prime}, x_{n}\right) \in \mathbb{R}^{n}, t>0$ from the following equations:

$$
\begin{gathered}
u_{t}-a(t) \Delta u=\int_{0}^{t} k\left(x^{\prime}, \tau\right) u(x, t-\tau) d \tau, \quad(x, t) \in \mathbb{R}_{T}^{n}, \\
\left.u\right|_{t=0}=\varphi(x), \quad x \in \mathbb{R}^{n}, \\
\left.u\right|_{x_{n}=0}=f\left(x^{\prime}, t\right), \quad 0 \leqslant t \leqslant T, \quad f\left(x^{\prime}, 0\right)=\varphi\left(x^{\prime}, 0\right),
\end{gathered}
$$

where $\triangle$ is the Laplace operator with respect to spatial variables $x=\left(x_{1}, \ldots, x_{n}\right)$, $\mathbb{R}_{T}^{n}=\left\{(x, t) \mid x=\left(x^{\prime}, x_{n}\right) \in \mathbb{R}^{n}, 0<t<T\right\}$ is a strip with thickness $T, T>0$ is an arbitrary fixed number; $a(t) \in C^{1}[0, T], 0<a_{0} \leqslant a(t) \leqslant a_{1}<\infty, a_{0}$ and $a_{1}$ are given numbers.

To the best of our knowledge, the problem $(0.1)-(0.3)$ have not been studied earlier. Our result generalizes the work [17] to the case of an integro-differential heat equation with a variable coefficient of thermal conductivity.

In the sequel,we will use the Hölder space $H^{\alpha}$ for functions depending on spatial variables and for functions depending on spatial and time variables - Hölder space $H^{\alpha, \alpha / 2}$ with exponents $\alpha$ and $\alpha / 2, \alpha$ is non-negative integer. Starting with Section 2, we will assume that $\varphi(x) \in H^{l+6}\left(\mathbb{R}^{n}\right)$, $\varphi(x) \geqslant \varphi_{0}=$ const $>0, f\left(x^{\prime}, t\right) \in H^{l+4,(l+4) / 2}\left(\overline{\mathbb{R}}_{T}^{n-1}\right)$,

$$
\overline{\mathbb{R}}_{T}^{n-1}=\left\{\left(x^{\prime}, t\right) \mid x^{\prime} \in \mathbb{R}^{n-1}, \quad 0 \leqslant t \leqslant T\right\}, \quad l \in(0,1),
$$

Spaces $H^{l}(Q), H^{l, l / 2}\left(Q_{T}\right)$ and norms in them are defined in [20, pp. 16-27]. In what follows, for norm of functions in the space $H^{l, l / 2}\left(Q_{T}\right)$ (in concrete cases $Q_{T}=\mathbb{R}_{T}^{n}$ or $Q_{T}=\mathbb{R}_{T}^{n-1}$ ) depending 
on spatial and time variables we will use notation $|\cdot|_{T}^{l, l / 2}$, while for functions depending only on spatial variables we will use $|\cdot|^{l}$ (in this case $Q=\mathbb{R}^{n}$ or $Q=\mathbb{R}^{n-1}$ ).

As a rule, inverse problems are ill-posed. According to [21, pp.31-44] problems that are ill-posed some spaces but can be well-posed for another choice of spaces, are called weakly illposed. In this work, functional spaces for given and unknown functions are indicated, in which the problem $(0.1)-(0.3)$ is well-posed. In this case, we restrict ourselves to proving the existence and uniqueness theorem for the solution of the problem (Section 4).

Note that the equation (0.1) is an integro-differential equation with a heat operator on the left and a Volterra-convolutional integral on the right sides. To get acquainted with the issues of solvability of various problems for integro-differential equations with Volterra-non-convolutional integrals, we refer the reader to the papers [22,23] (also see the literature in them).

The article is organized as follows. In Section 1, we investigate the direct problem $(0.1)$ and (0.2). In Section 2, we transform the given problem into an auxiliary problem where the additional condition contains the unknown $k$ outside integral. In Section 3, we reduce the auxiliary problem to a system of integral equations with respect to unknown functions. In Section 4, we prove the main result which states the existence and uniqueness of solution of the given problem by a fixed point argument.

\section{$\S 1$. Direct problem}

For the given functions $a$ and $k$, the problem of determining the solution to the integrodifferential equation (0.1) under the initial condition (0.2) is the Cauchy problem. This problem in the theory of inverse problems is called the direct problem.

The solution of the Cauchy problem $(0.1)$ and $(0.2)$ can be reduced to the solution of an integral equation of the Volterra type. To do this we use the formula

$$
\begin{gathered}
p(x, t)=\int_{\mathbb{R}^{n}} \varphi(\xi) G(x-\xi ; \theta(t)) d \xi+ \\
+\int_{0}^{\theta(t)} \frac{d \tau}{a\left(\theta^{-1}(\tau)\right)} \int_{\mathbb{R}^{n}} F\left(\xi, \theta^{-1}(\tau)\right) G(x-\xi ; \theta(t)-\tau) d \xi
\end{gathered}
$$

which provides the solution of the following Cauchy problem for the heat equation with timevariable thermal conductivity:

$$
\begin{gathered}
p_{t}-a(t) \Delta p=F(x, t), \quad x \in \mathbb{R}^{n}, \quad t>0, \\
p(x, 0)=\varphi(x), \quad x \in \mathbb{R}^{n} .
\end{gathered}
$$

In (1.1) $\theta(t)=\int_{0}^{t} a(\tau) d \tau$ and $\theta^{-1}(t)$ is the inverse function to $\theta(t)$;

$$
G(x-\xi ; \theta(t)-\tau)=\frac{1}{(2 \sqrt{\pi(\theta(t)-\tau)})^{n}} e^{\frac{-|x-\xi|^{2}}{4(\theta(t)-\tau)}}
$$

is the fundamental solution of the heat operator with the time-dependent coefficient of thermal conductivity $\frac{\partial}{\partial t}-a(t) \triangle, \xi=\left(\xi_{1}, \ldots, \xi_{n}\right), \xi^{\prime}=\left(\xi_{1}, \ldots, \xi_{n-1}\right), \quad d \xi=d \xi_{1} \ldots d \xi_{n},|x|^{2}=x_{1}^{2}+$ $+\ldots+x_{n}^{2}$.

Taking this into account, we obtain the following integral equation to determine $u(x, t)$ :

$$
\begin{gathered}
u(x, t)=\int_{\mathbb{R}^{n}} \varphi(\xi) G(x-\xi ; \theta(t)) d \xi+ \\
+\int_{0}^{\theta(t)} \frac{d \tau}{a\left(\theta^{-1}(\tau)\right)} \int_{\mathbb{R}^{n}} \int_{0}^{\theta^{-1}(\tau)} k\left(\xi^{\prime}, \alpha\right) u\left(\xi, \theta^{-1}(\tau)-\alpha\right) G(x-\xi ; \theta(t)-\tau) d \alpha d \xi
\end{gathered}
$$


Lemma 1. If $\varphi(x) \in H^{l+2}\left(\mathbb{R}^{n}\right), k\left(x^{\prime}, t\right) \in H^{l, l / 2}\left(\overline{\mathbb{R}}_{T}^{n-1}\right)$, and a $(t)$ satisfies the conditions of Introduction, then there exists a unique solution of integral equation (1.2) in the space $u(x, t) \in$ $\in H^{l+2,(l+2) / 2}\left(\mathbb{R}_{T}^{n}\right)$.

P r o o f. To proof Lemma we use the method of successive approximations and consider the sequence of functions defined recursively by the formulas:

$$
\begin{gathered}
u_{0}(x, t)=\int_{\mathbb{R}^{n}} \varphi(\xi) G(x-\xi ; \theta(t)) d \xi, \\
u_{n}(x, t)= \\
=\int_{0}^{\theta(t)} \frac{d \tau}{a\left(\theta^{-1}(\tau)\right)} \int_{\mathbb{R}^{n}} \int_{0}^{\theta^{-1}(\tau)} k\left(\xi^{\prime}, \alpha\right) u_{n-1}\left(\xi, \theta^{-1}(\tau)-\alpha\right) G(x-\xi ; \theta(t)-\tau) d \alpha d \xi, \\
(x, t) \in \mathbb{R}_{T}^{n}, \quad n=1,2, \ldots
\end{gathered}
$$

Under the assumptions of Lemma, one has the inclusion $u(x, t) \in H^{l+2,(l+2) / 2}\left(\mathbb{R}_{T}^{n}\right)$. According to the general theory of Cauchy problems for equations of the parabolic type (see, [20]), this implies that the same property will be possessed by all functions $u_{n}(x, t)$ in $\mathbb{R}_{T}^{n}$ defined by relations (1.3). Set $\varphi_{0}=|\varphi(x)|^{l+2}$. Using (1.3), we estimate the modulus of $u_{n}(x, t)$ in the domain $\mathbb{R}_{T}^{n}$ as

$$
\begin{gathered}
\left|u_{0}(x, t)\right|_{T}^{l+2,(l+2) / 2} \leqslant \varphi_{0}, \\
\left|u_{1}(x, t)\right|_{T}^{l+2,(l+2) / 2} \leqslant \int_{0}^{\theta(t)} \frac{d \tau}{a\left(\theta^{-1}(\tau)\right)} \int_{\mathbb{R}^{n}} \int_{0}^{\theta^{-1}(\tau)}\left|k\left(\xi^{\prime}, \alpha\right)\right|_{T}^{l, l / 2}\left|u_{0}\left(\xi, \theta^{-1}(\tau)-\alpha\right)\right|_{T}^{l+2,(l+2) / 2} \times \\
\times G(x-\xi ; \theta(t)-\tau) d \alpha d \xi \leqslant \varphi_{0} \frac{a_{1} k_{0} T}{a_{0}} \frac{t}{1 !}, \quad(x, t) \in \mathbb{R}_{T}^{n}, \quad k_{0}=\left|k\left(x^{\prime}, t\right)\right|_{T}^{l, l / 2},
\end{gathered}
$$

for arbitrary $n \geqslant 1$, we have

$$
\left|u_{n}(x, t)\right|_{T}^{l+2,(l+2) / 2} \leqslant \varphi_{0}\left(\frac{a_{1} k_{0} T}{a_{0}}\right)^{n} \frac{t^{n}}{n !} .
$$

In last estimates we have used the relation $\int_{\mathbb{R}^{n}} G(x-\xi, \theta(t)-\tau) d \xi=1$. It follows from the above estimates that the series $\sum_{n=1}^{\infty} u_{n}(x, t)$ converges in $\mathbb{R}_{T}^{n}$ and its sum $u(x, t)$ belongs to the function space $H^{l+2,(l+2) / 2}$. Since the sequence $u_{n}(x, t)$ defined by relations (1.3) converges to $u(x, t)$ uniformly in $\mathbb{R}_{T}^{n}$, we conclude that $u(x, t)$ is a solution of equation (1.2).

Now we show that this equation has a unique solution. Assume that there exist two solutions $u^{1}(x, t)$ and $u^{2}(x, t)$ of equation (1.2). Then their difference $Z(x, t)=u^{2}(x, t)-u^{1}(x, t)$ is a solution of the equation

$$
Z(x, t)=\int_{0}^{\theta(t)} \frac{d \tau}{a\left(\theta^{-1}(\tau)\right)} \int_{\mathbb{R}^{n}} \int_{0}^{\theta^{-1}(\tau)} k\left(\xi^{\prime}, \alpha\right) Z\left(\xi, \theta^{-1}(\tau)-\alpha\right) G(x-\xi, \theta(t)-\tau) d \alpha d \xi
$$

Denote by $\tilde{Z}(t)$ the supremum of the modulus of the function $Z(x, t)$ over $x \in \mathbb{R}^{n}$ for each fixed $t \in[0, T]$. Then we have the inequality

$$
\tilde{Z}(t) \leqslant \frac{a_{1} k_{0} T}{a_{0}} \int_{0}^{a_{1} t} \tilde{Z}(\tau) d \tau, \quad t \in[0, T] .
$$

It is well known that there can be only one solution to this integral inequality: $\tilde{Z}(t)=0$ for $t \in[0, T]$. Hence we also have $Z(x, t)=0$ in $\mathbb{R}_{T}^{n}$; that is, $u^{1}(x, t)=u^{2}(x, t)$ in $\mathbb{R}_{T}^{n}$. Consequently, the equation (1.2) has a unique solution in $\mathbb{R}_{T}^{n}$. The proof of the lemma is complete. 


\section{§2. Auxiliary problems}

We introduce new function $\vartheta(x, t)$ by formula $\vartheta(x, t)=u_{x_{n} x_{n}}(x, t)$. Then the problem $(0.1)-$ (0.3) leads us to the following relations:

$$
\begin{gathered}
\vartheta_{t}-a(t) \Delta \vartheta=\int_{0}^{t} k\left(x^{\prime}, \tau\right) \vartheta(x, t-\tau) d \tau \\
\left.\vartheta\right|_{t=0}=\varphi_{x_{n} x_{n}}(x) .
\end{gathered}
$$

From the equalities $(0.1)$ and (0.2) we obtain the additional condition for $\vartheta(x, t)$ by separating $a(t) u_{x_{n} x_{n}}$ in the term $a(t) \Delta u$ of $(0.1)$ at $x_{n}=0$ and using (2.2):

$$
\left.\vartheta\right|_{x_{n}=0}=\frac{1}{a(t)} f_{t}-\sum_{i=1}^{n-1} \frac{\partial^{2}}{\partial x_{i}^{2}} f-\frac{1}{a(t)} \int_{0}^{t} k\left(x^{\prime}, \tau\right) f\left(x^{\prime}, t-\tau\right) d \tau,
$$

From this equality and (2.2) the matching condition of the given functions follows

$$
\varphi_{x_{n} x_{n}}\left(x^{\prime}, 0\right)=\frac{1}{a(0)} f_{t}\left(x^{\prime}, 0\right)-\sum_{i=1}^{n-1} f_{x_{i} x_{i}}\left(x^{\prime}, 0\right) .
$$

Now differentiate the equation (2.1) and (2.3) with respect to $t$ and denote $\vartheta_{t}(x, t)=\omega(x, t)$. As result, one has problem of finding functions $\vartheta(x, t), k\left(x^{\prime}, t\right), \omega(x, t)$ from (2.1), (2.2) and the following equations:

$$
\begin{gathered}
\omega_{t}-a(t) \Delta \omega=(\ln a(t))^{\prime} \omega-(\ln a(t))^{\prime} \int_{0}^{t} k\left(x^{\prime}, \tau\right) \vartheta(x, t-\tau) d \tau+ \\
+\int_{0}^{t} k\left(x^{\prime}, \tau\right) \omega(x, t-\tau) d \tau+k\left(x^{\prime}, t\right) \varphi_{x_{n} x_{n}}(x), \\
\left.\omega\right|_{t=0}=a(0) \Delta \varphi_{x_{n} x_{n}}(x), \\
\left.\omega\right|_{x_{n}=0}=-\frac{a^{\prime}(t)}{a^{2}(t)} f_{t}+\frac{1}{a(t)} f_{t t}-\sum_{k=1}^{n-1} \frac{\partial^{2}}{\partial x_{k}^{2}} f_{t}+\frac{a^{\prime}(t)}{a^{2}(t)} \int_{0}^{t} k\left(x^{\prime}, \tau\right) f\left(x^{\prime}, t-\tau\right) d \tau- \\
-\frac{1}{a(t)} \int_{0}^{t} k\left(x^{\prime}, \tau\right) f_{t}\left(x^{\prime}, t-\tau\right) d \tau-\frac{1}{a(t)} k\left(x^{\prime}, t\right) \varphi\left(x^{\prime}, 0\right)
\end{gathered}
$$

Here the initial condition was obtained by setting in (2.1) $t=0$ and using (2.2). Note that the function $k\left(x^{\prime}, t\right)$ enters into the equation (2.7) without integral.

It is not difficult to show that at fulfilling of the matching conditions in (0.3), (2.4) and smoothness of given functions $\varphi, f$ the inverse transformations are took place [17].

Thus, we proved the following assertion:

Lemma 2. Suppose that all the conditions for the functions $a, \varphi$ and $f$ made in introduction are satisfied. Moreover, the matching conditions in (0.3) and (2.4) hold. Then, the problem (0.1)(0.3) is equivalent to the auxiliary problems of determining functions $\vartheta(x, t), k\left(x^{\prime}, t\right), \omega(x, t)$ from (2.1), (1.2) and (2.5)-(2.7).

\section{$\S 3$. Reduction of the Auxiliary problems to the integral equations}

Here we prove the main result of this Section, which is the following assertion:

Lemma 3. The auxiliary problem (2.1), (2.2) and (2.5)-(2.7) is equivalent to the problem of determining functions $\vartheta(x, t), k\left(x^{\prime}, t\right), \omega(x, t)$ from the following system of integral equations: 


$$
\begin{aligned}
& \vartheta(x, t)=\int_{\mathbb{R}^{n}} \varphi_{\xi_{n} \xi_{n}}(\xi) G(x-\xi, \theta(t)) d \xi+ \\
& +\int_{0}^{\theta(t)} \frac{d \tau}{a\left(\theta^{-1}(\tau)\right)} \int_{\mathbb{R}^{n}} \int_{0}^{\theta^{-1}(\tau)} k\left(\xi^{\prime}, \alpha\right) \vartheta\left(\xi, \theta^{-1}(\tau)-\alpha\right) G(x-\xi, \theta(t)-\tau) d \alpha d \xi, \\
& \omega(x, t)=\int_{\mathbb{R}^{n}} a(0) \Delta \varphi_{\xi_{n} \xi_{n}}(\xi) G(x-\xi, \theta(t)) d \xi+ \\
& +\int_{0}^{\theta(t)} \frac{d \tau}{a\left(\theta^{-1}(\tau)\right)} \int_{\mathbb{R}^{n}}\left[\left(\ln a\left(\theta^{-1}(\tau)\right)\right)^{\prime} \omega\left(\xi, \theta^{-1}(\tau)\right)-\right. \\
& -\left(\ln a\left(\theta^{-1}(\tau)\right)\right)^{\prime} \int_{0}^{\theta^{-1}(\tau)} k\left(\xi^{\prime}, \alpha\right) \vartheta\left(\xi, \theta^{-1}(\tau)-\alpha\right) d \alpha+ \\
& \left.+\int_{0}^{\theta^{-1}(\tau)} k\left(\xi^{\prime}, \alpha\right) \omega\left(\xi, \theta^{-1}(\tau)-\alpha\right) d \alpha+k\left(\xi^{\prime}, \theta^{-1}(\tau)\right) \varphi_{\xi_{n} \xi_{n}}(\xi)\right] G(x-\xi, \theta(t)-\tau) d \xi, \\
& k\left(x^{\prime}, t\right)=\frac{a(t)}{\varphi\left(x^{\prime}, 0\right)}\left[-\int_{R^{n}} a(0) \Delta \varphi_{\xi_{n} \xi_{n}}(\xi) G\left(x^{\prime}-\xi^{\prime}, \xi_{n}, \theta(t)\right) d \xi-\right. \\
& \left.-\frac{a^{\prime}(t)}{a^{2}(t)} f_{t}+\frac{1}{a(t)} f_{t t}-\sum_{k=1}^{n-1} \frac{\partial^{2}}{\partial x_{k}^{2}} f_{t}\right]+ \\
& +\frac{a(t)}{\varphi\left(x^{\prime}, 0\right)}\left(-\int_{0}^{\theta(t)} \frac{d \tau}{a\left(\theta^{-1}(\tau)\right)} \int_{R^{n}}\left[\left(\ln a\left(\theta^{-1}(\tau)\right)\right)^{\prime} \omega\left(\xi, \theta^{-1}(\tau)\right)-\right.\right. \\
& -\left(\ln a\left(\theta^{-1}(\tau)\right)^{\prime} \int_{0}^{\theta^{-1}(\tau)} k\left(\xi^{\prime}, \alpha\right) \vartheta\left(\xi, \theta^{-1}(\tau)-\alpha\right) d \alpha+\right. \\
& +\int_{0}^{\theta^{-1}(\tau)} k\left(\xi^{\prime}, \alpha\right) \omega\left(\xi, \theta^{-1}(\tau)-\alpha\right) d \alpha+ \\
& \left.+k\left(\xi^{\prime}, \theta^{-1}(\tau)\right) \varphi_{\xi_{n} \xi_{n}}(\xi)\right] G\left(x^{\prime}-\xi^{\prime}, \xi_{n}, \theta(t)-\tau\right) d \xi+ \\
& \left.+\frac{a^{\prime}(t)}{a^{2}(t)} \int_{0}^{t} k\left(x^{\prime}, \tau\right) f\left(x^{\prime}, t-\tau\right) d \tau-\frac{1}{a(t)} \int_{0}^{t} k\left(x^{\prime}, \tau\right) f_{t}\left(x^{\prime}, t-\tau\right) d \tau\right) .
\end{aligned}
$$

P r o o f. To prove Lemma it is not difficult to see that in view of (1.1) the integral equations (3.1), (3.2) are obtained from the problems (2.1), (2.2) and (2.5), (2.6), respectively. The equation (3.3) follows from (3.2) taking into account (2.7).

\section{§4. Existence and uniqueness}

This section contains the main result of this paper. Here the existence and uniqueness result for the problem (2.1)-(2.3) is proved using the contraction mapping principle [24, pp. 87-97]. The idea is to write the integral equations for unknown functions $\vartheta(x, t), \omega(x, t), k\left(x^{\prime}, t\right)$ as a system with a nonlinear operator, and prove that this operator is a contraction mapping operator for sufficiently small $T$. The existence and uniqueness result then follows immediately.

Definition 1. Let $F$ be an operator defined on a closed set $\Omega$ which is a subset of a Banach space. $F$ is called a contraction mapping operator in $\Omega$ if it satisfies the following two properties:

(1) if $y \in \Omega$, then $F x \in \Omega$ (that is $F$ maps $\Omega$ into itself);

(2) if $y, z \in \Omega$, then $\|F y-F z\| \leqslant \rho\|y-z\|$ with $\rho<1$ ( $\rho-$ is a constant independent of $y$ and $z)$. 
Lemma 4 (contraction mapping principle [24, pp.87-97]). If $F$ is a contraction mapping operator from $\Omega$ to $\Omega$, then the equation $y=F y$ has a unique solution $y_{0} \in \Omega$.

Theorem 1 (existence and uniqueness). Suppose that all the conditions for the functions $a, \varphi$ and $f$ made in Introduction are satisfied. Moreover, the matching conditions in (0.3) and (2.4) are met. Then there exists a sufficiently small number $T>0$ that the solution to the integral equations (3.1)-(3.3) in the class of functions $\{\vartheta(x, t), \omega(x, t)\} \in H^{l+2,(l+2) / 2}\left(\overline{\mathbb{R}}_{T}^{n}\right), k\left(x^{\prime}, t\right) \in H^{l, l / 2}\left(\overline{\mathbb{R}}_{T}^{n-1}\right)$ exists and is unique. Thus, there is the unique classical solution to the problem (0.1)-(0.3).

P r o o f. The system of equations (3.1), (3.2), (3.3) is a closed system for the unknown functions $\vartheta(x, t), \omega(x, t), k\left(x^{\prime}, t\right)$ in the domain $\mathbb{R}_{T}^{n}$. It can be rewritten in a nonlinear operator equation

$$
\psi=A \psi
$$

where $\psi=\left(\psi_{1}, \psi_{2}, \psi_{3}\right)^{*}=\left(\vartheta(x, t), \omega(x, t), k\left(x^{\prime}, t\right)\right)^{*}, *$ is the symbol of transposition, and according to the right sides of the equations (3.1)-(3.3), operator $A$ has the form:

$A \psi=\left[(A \psi)_{1},(A \psi)_{2},(A \psi)_{3}\right]$, where

$$
\begin{aligned}
& (A \psi)_{1}=\psi_{01}(x, t)+ \\
& +\int_{0}^{\theta(t)} \frac{d \tau}{a\left(\theta^{-1}(\tau)\right)} \int_{\mathbb{R}^{n}} \int_{0}^{\theta^{-1}(\tau)} \psi_{3}\left(\xi^{\prime}, \alpha\right) \psi_{1}\left(\xi, \theta^{-1}(\tau)-\alpha\right) G(x-\xi, \theta(t)-\tau) d \alpha d \xi \\
& (A \psi)_{2}=\psi_{02}(x, t)+\int_{0}^{\theta(t)} \frac{d \tau}{a\left(\theta^{-1}(\tau)\right)} \int_{\mathbb{R}^{n}}\left[\left(\ln a\left(\theta^{-1}(\tau)\right)^{\prime} \psi_{2}\left(\xi, \theta^{-1}(\tau)\right)-\right.\right. \\
& -\left(\ln a\left(\theta^{-1}(\tau)\right)^{\prime} \int_{0}^{\theta^{-1}(\tau)} \psi_{3}\left(\xi^{\prime}, \alpha\right) \psi_{1}(\xi, \tau-\alpha) d \alpha+\right. \\
& +\int_{0}^{\theta^{-1}(\tau)} \psi_{3}\left(\xi^{\prime}, \alpha\right) \psi_{2}\left(\xi, \theta^{-1}(\tau)-\alpha\right) d \alpha+ \\
& \left.+\psi_{3}\left(\xi^{\prime}, \theta^{-1}(\tau)\right) \varphi_{\xi_{n} \xi_{n}}(\xi)\right] G(x-\xi, \theta(t)-\tau) d \xi, \\
& (A \psi)_{3}=\psi_{03}\left(x^{\prime}, t\right)+\frac{a(t)}{\varphi\left(x^{\prime}, 0\right)}\left(-\int_{0}^{\theta(t)} \frac{d \tau}{a\left(\theta^{-1}(\tau)\right)} \int_{\mathbb{R}^{n}}\left[\left(\ln a\left(\theta^{-1}(\tau)\right)\right)^{\prime} \psi_{2}\left(\xi, \theta^{-1}(\tau)\right)-\right.\right. \\
& -\left(\ln a\left(\theta^{-1}(\tau)\right)\right)^{\prime} \int_{0}^{\theta^{-1}(\tau)} \psi_{3}\left(\xi^{\prime}, \alpha\right) \psi_{1}\left(\xi, \theta^{-1}(\tau)-\alpha\right) d \alpha+ \\
& +\int_{0}^{\theta^{-1}(\tau)} \psi_{3}\left(\xi^{\prime}, \alpha\right) \psi_{2}\left(\xi, \theta^{-1}(\tau)-\alpha\right) d \alpha+ \\
& \left.\left.+\psi_{3}\left(\xi^{\prime}, \theta^{-1}(\tau)\right) \varphi_{\xi_{n} \xi_{n}}(\xi)\right] G\left(x^{\prime}-\xi^{\prime}, \xi_{n}, \theta(t)-\tau\right) d \xi\right)+ \\
& +\frac{(\ln a(t))^{\prime}}{\varphi\left(x^{\prime}, 0\right)} \int_{0}^{t} \psi_{3}\left(x^{\prime}, \tau\right) f\left(x^{\prime}, t-\tau\right) d \tau-\frac{1}{\varphi\left(x^{\prime}, 0\right)} \int_{0}^{t} \psi_{3}\left(x^{\prime}, \tau\right) f_{t}\left(x^{\prime}, t-\tau\right) d \tau .
\end{aligned}
$$

In (4.2)-(4.4) we introduced notations:

$$
\begin{gathered}
\psi_{01}(x, t)=\int_{\mathbb{R}^{n}} \varphi_{\xi_{n} \xi_{n}}(\xi) G(x-\xi, \theta(t)) d \xi \\
\psi_{02}(x, t)=\int_{\mathbb{R}^{n}} a(0) \Delta \varphi_{\xi_{n} \xi_{n}}(\xi) G\left(x^{\prime}-\xi^{\prime}, \xi_{n}, \theta(t)\right) d \xi \\
\psi_{03}\left(x^{\prime}, t\right)= \\
=\frac{a(t)}{\varphi\left(x^{\prime}, 0\right)}\left[-\int_{\mathbb{R}^{n}} a(0) \Delta \varphi_{\xi_{n} \xi_{n}}(\xi) G\left(x^{\prime}-\xi^{\prime}, \xi_{n}, \theta(t)\right) d \xi-\frac{a^{\prime}(t)}{a^{2}(t)} f_{t}+\frac{1}{a(t)} f_{t t}-\sum_{k=1}^{n-1} \frac{\partial^{2}}{\partial x_{k}^{2}} f_{t}\right] .
\end{gathered}
$$


Denote $|\psi|_{T}^{l}=\max \left(\left|\psi_{1}\right|_{T}^{l / 2},\left|\psi_{2}\right|_{T}^{l / 2},\left|\psi_{3}\right|_{T}^{l / 2}\right), T<T_{0}, T_{0}$ is some positive number and consider in the space $H^{l, l / 2}\left(\mathbb{R}_{T}^{n}\right)$ the set $S(T)$ of functions $\psi(x, t)$, which obey the inequality

$$
\left|\psi-\psi_{0}\right|_{T}^{l} \leqslant\left|\psi_{0}\right|_{T_{0}}^{l},
$$

where $\psi_{0}=\left(\psi_{01}, \psi_{02}, \psi_{03}\right)$ and $\left|\psi_{0}\right|_{T_{0}}^{l}=\max \left(\left|\psi_{01}\right|_{T_{0}}^{l},\left|\psi_{02}\right|_{T_{0}}^{l},\left|\psi_{03}\right|_{T_{0}}^{l}\right)$.

It can be demonstrated that $A$ satisfies the conditions of contraction mapping operator in $S(T)$, if $T_{0}$ is sufficiently small. Let $\psi \in S(T), T<T_{0}$. Then from the inequality (4.5), we have

$$
\left|\psi_{i}\right|_{T}^{l} \leqslant 2\left|\psi_{0}\right|_{T_{0}}^{l}, \quad i=1,2,3 .
$$

The Cauchy problem setting up the heat conduction equation has a solution, if initial condition, e.g., $\varphi$ function is belong to the class $H^{l+2}$ [20, p. 364]. In our case, $\varphi$ is in $H^{l+6}$, because its fourth order derivatives are involved in the auxiliary problem.

Let us introduce the notations:

$$
a_{2}:=\max _{t \in[0, T]}\left|(\ln a(t))^{\prime}\right|, \quad \varphi_{1}:=|\varphi|^{l+6}, \quad f_{0}:=|f|_{T}^{l+4,(l+4) / 2} .
$$

First it is shown that $A$ has the first property of a contraction mapping operator. Using the estimates of the thermal volume potentials [20, pp.318-325] it is easy to obtain the following inequalities:

$$
\begin{gathered}
\left|(A \psi)_{1}-\psi_{01}\right|_{T}^{l}= \\
=\left|\int_{0}^{\theta(t)} \frac{d \tau}{a\left(\theta^{-1}(\tau)\right)} \int_{\mathbb{R}^{n}} \int_{0}^{\theta^{-1}(\tau)} \psi_{3}\left(\xi^{\prime}, \alpha\right) \psi_{1}\left(\xi, \theta^{-1}(\tau)-\alpha\right) G(x-\xi, \theta(t)-\tau) d \alpha d \xi\right|_{T}^{l} \leqslant \\
\leqslant 4 \beta_{0}(T) a_{0}^{-1}\left(\left|\psi_{0}\right|_{T_{0}}^{l}\right)^{2}, \\
\left|(A \psi)_{2}-\psi_{02}\right|_{T}^{l}=\mid \int_{0}^{\theta(t)} \frac{d \tau}{a\left(\theta^{-1}(\tau)\right)} \int_{\mathbb{R}^{n}}\left[\left(\ln a\left(\theta^{-1}(\tau)\right)\right)^{\prime} \psi_{2}\left(\xi, \theta^{-1}(\tau)\right)-\right. \\
-\left(\ln a\left(\theta^{-1}(\tau)\right)\right)^{\prime} \int_{0}^{\theta^{-1}(\tau)} \psi_{3}\left(\xi^{\prime}, \alpha\right) \psi_{1}\left(\xi, \theta^{-1}(\tau)-\alpha\right) d \alpha+ \\
\left.+\int_{0}^{\theta^{-1}(\tau)} \psi_{3}\left(\xi^{\prime}, \alpha\right) \psi_{2}\left(\xi, \theta^{-1}(\tau)-\alpha\right) d \alpha+\psi_{3}\left(\xi^{\prime}, \tau\right) \varphi_{\xi_{n} \xi_{n}}(\xi)\right]\left.G(x-\xi, \theta(t)-\tau) d \xi\right|_{T} ^{l} \leqslant \\
\leqslant 2 \beta_{1}(T) a_{0}^{-1}\left(a_{2}+\varphi_{1}\right)\left|\psi_{0}\right|_{T_{0}}^{l}+4 \beta_{2}(T) a_{0}^{-1}\left(a_{2}+1\right)\left(\left|\psi_{0}\right|_{T_{0}}^{l}\right)^{2}, \\
\left|(A \psi)_{3}-\psi_{03}\right|_{T}^{l}=\mid \frac{a(t)}{\varphi\left(x^{\prime}, 0\right)}\left(-\int_{0}^{\theta(t)} \frac{d \tau}{a\left(\theta^{-1}(\tau)\right)} \int_{\mathbb{R}^{n}}\left[\left(\ln a\left(\theta^{-1}(\tau)\right)\right)^{\prime} \psi_{2}\left(\xi, \theta^{-1}(\tau)\right)-\right.\right. \\
+\left(\ln a\left(\theta^{-1}(\tau)\right)\right)^{\prime} \int_{0}^{\theta^{-1}(\tau)} \psi_{3}\left(\xi^{\prime}, \alpha\right) \psi_{1}\left(\xi, \theta^{-1}(\tau)-\alpha\right) d \alpha+ \\
\left.\left.+\int_{0}^{\theta^{-1}(\tau)} \psi_{3}\left(\xi^{\prime}, \alpha\right) \psi_{2}\left(\xi, \theta^{-1}(\tau)-\alpha\right) d \alpha+\psi_{3}\left(\xi^{\prime}, \theta^{-1}(\tau)\right) \varphi_{\xi_{n} \xi_{n}}(\xi)\right] G\left(x^{\prime}-\xi^{\prime}, \xi_{n}, \theta(t)-\tau\right) d \xi\right)+ \\
+\frac{(\ln a(t))^{\prime}}{\varphi\left(x^{\prime}, 0\right)} \int_{0}^{t} \psi_{3}\left(x^{\prime}, \tau\right) f\left(x^{\prime}, t-\tau\right) d \tau-\left.\frac{1}{\varphi\left(x^{\prime}, 0\right)} \int_{0}^{t} \psi_{3}\left(x^{\prime}, \tau\right) f_{t}\left(x^{\prime}, t-\tau\right) d \tau\right|_{T} ^{l} \leqslant \\
\leqslant 2\left[\beta_{1}(T) a_{1} a_{0}^{-1} \varphi_{0}^{-1}\left(a_{2}+\varphi_{1}\right)+f_{0} \varphi_{0}^{-1} T_{0}\left(a_{2}+1\right)\right]\left|\psi_{0}\right|_{T_{0}}^{l}+4 \beta_{2}(T) a_{1} a_{0}^{-1} \varphi_{0}^{-1}\left(a_{2}+1\right)\left(\left|\psi_{0}\right|_{T_{0}}^{l}\right)^{2}
\end{gathered}
$$

where $\beta_{i}(T)$ are increasing functions of $T$ and $\beta_{i}(T) \rightarrow 0$ at $T \rightarrow 0$ for all $i=0,1,2$. Therefore, if we choose $T_{0}$ so that the following inequalities should be satisfied:

$$
\begin{gathered}
4 \beta_{0}(T) a_{0}^{-1}\left|\psi_{0}\right|_{T_{0}}^{l} \leqslant 1, \\
2 \beta_{1}(T) a_{0}^{-1}\left(a_{2}+\varphi_{1}\right)+4 \beta_{2}(T) a_{0}^{-1}\left(a_{2}+1\right)\left|\psi_{0}\right|_{T_{0}}^{l} \leqslant 1, \\
2\left[\beta_{1}(T) a_{1} a_{0}^{-1} \varphi_{0}^{-1}\left(a_{2}+\varphi_{1}\right)+f_{0} \varphi_{0}^{-1} T_{0}\left(a_{2}+1\right)\right]+4 \beta_{2}(T) a_{1} a_{0}^{-1} \varphi_{0}^{-1}\left(a_{2}+1\right)\left|\psi_{0}\right|_{T_{0}}^{l} \leqslant 1,
\end{gathered}
$$


then the operator $A$ for $T<T_{0}$ has the first property of a contraction mapping operator, that is, $A \psi \in S(T)$.

Consider next the second property of a contraction mapping operator for $A$. Let $\psi^{(1)}=$ $=\left(\psi_{1}^{(1)}, \psi_{2}^{(1)}, \psi_{3}^{(1)}\right) \in S(T), \psi^{(2)}=\left(\psi_{1}^{(2)}, \psi_{2}^{(2)}, \psi_{3}^{(2)}\right) \in S(T)$. Then we have

$$
\begin{gathered}
\left.\mid\left((A \psi)^{(1)}-A \psi\right)^{(2)}\right)\left._{1}\right|_{T} ^{l}=\mid \int_{0}^{\theta(t)} \frac{d \tau}{a\left(\theta^{-1}(\tau)\right)} \int_{\mathbb{R}^{n}} \int_{0}^{\theta^{-1}(\tau)}\left[\psi_{3}^{(1)}\left(\xi^{\prime}, \alpha\right) \psi_{1}^{(1)}\left(\xi, \theta^{-1}(\tau)-\alpha\right)-\right. \\
\left.-\psi_{3}^{(2)}\left(\xi^{\prime}, \alpha\right) \psi_{1}^{(2)}\left(\xi, \theta^{-1}(\tau)-\alpha\right)\right]\left.G(x-\xi, \theta(t)-\tau) d \alpha d \xi\right|_{T} ^{l} .
\end{gathered}
$$

Here the integrand in the last integral can be estimated as follows

$$
\begin{gathered}
\left|\psi_{3}^{(1)} \psi_{1}^{(1)}-\psi_{3}^{(2)} \psi_{1}^{(2)}\right|_{T}^{l}=\left|\left(\psi_{3}^{(1)}-\psi_{2}^{(2)}\right) \psi_{1}^{(1)}+\psi_{3}^{(2)}\left(\psi_{1}^{(1)}-\psi_{1}^{(2)}\right)\right|_{T}^{l} \leqslant \\
\leqslant 2\left|\psi^{(1)}-\psi^{(2)}\right|_{T}^{l} \max \left(\left|\psi_{1}^{(1)}\right|_{T}^{l},\left|\psi_{3}^{(2)}\right|_{T}^{l}\right) \leqslant 4\left|\psi_{0}\right|_{T}^{l}\left|\psi^{(1)}-\psi^{(2)}\right|_{T}^{l} .
\end{gathered}
$$

Therefore

$$
\left|\left(A \psi^{(1)}-A \psi^{(2)}\right)_{1}\right|_{T}^{l} \leqslant 8 \beta_{0}(T) a_{0}^{-1}\left|\psi_{0}\right|_{T_{0}}^{l}\left|\psi^{(1)}-\psi^{(2)}\right|_{T_{0}}^{l}
$$

The second and third components of $A \psi$ can be estimated in the analogous way:

$$
\begin{gathered}
\left|\left(A \psi^{(1)}-A \psi^{(2)}\right)_{2}\right|_{T}^{l}= \\
=\mid \int_{0}^{\theta(t)} \frac{d \tau}{a\left(\theta^{-1}(\tau)\right)} \int_{\mathbb{R}^{n}}\left[\left(\ln a\left(\theta^{-1}(\tau)\right)\right)^{\prime}\left(\psi_{2}^{(1)}\left(\xi, \theta^{-1}(\tau)\right)-\psi_{2}^{(2)}\left(\xi, \theta^{-1}(\tau)\right)\right)-\right. \\
-\left(\ln a\left(\theta^{-1}(\tau)\right)\right)^{\prime} \int_{0}^{\theta^{-1}(\tau)}\left(\psi_{3}^{(1)}\left(\xi^{\prime}, \alpha\right) \psi_{1}^{(1)}\left(\xi, \theta^{-1}(\tau)-\alpha\right)-\right. \\
\left.-\psi_{3}^{(2)}\left(\xi^{\prime}, \alpha\right) \psi_{1}^{(2)}\left(\xi, \theta^{-1}(\tau)-\alpha\right)\right) d \alpha+ \\
+\int_{0}^{\theta^{-1}(\tau)}\left(\psi_{3}^{(1)}\left(\xi^{\prime}, \alpha\right) \psi_{2}^{(1)}\left(\xi, \theta^{-1}(\tau)-\alpha\right)-\psi_{3}^{(2)}\left(\xi^{\prime}, \alpha\right) \psi_{2}^{(2)}\left(\xi, \theta^{-1}(\tau)-\alpha\right)\right) d \alpha+ \\
\left.+\left(\psi_{3}^{(1)}\left(\xi^{\prime}, \theta^{-1}(\tau)\right)-\psi_{3}^{(2)}\left(\xi^{\prime}, \theta^{-1}(\tau)\right)\right) \varphi_{\xi_{n} \xi_{n}}(\xi)\right]\left.G(x-\xi, \theta(t)-\tau) d \xi\right|_{T_{0}} ^{l} \leqslant \\
\leqslant\left[2 \beta_{1}(T) a_{0}^{-1}\left(a_{2}+\varphi_{1}\right)+8 \beta_{2}(T) a_{0}^{-1}\left(a_{2}+1\right)\left|\psi_{0}\right|_{T_{0}}^{l}\right]\left|\psi^{(1)}-\psi^{(2)}\right|_{T_{0}}^{l} . \\
=\mid \begin{array}{c}
a(t) \\
\varphi\left(x^{\prime}, 0\right)
\end{array}-\int_{0}^{\theta(t)} \frac{d \tau}{a\left(\theta^{-1}(\tau)\right)} \int_{\mathbb{R}^{n}}\left[\left(\ln a\left(\theta^{-1}(\tau)\right)\right)^{\prime}\left(\psi_{2}^{(1)}\left(\xi, \theta^{-1}(\tau)\right)-\psi_{2}^{(2)}\left(\xi, \theta^{-1}(\tau)\right)\right)-\right. \\
-\left(\ln a\left(\theta^{-1}(\tau)\right)\right)^{\prime} \int_{0}^{\theta^{-1}(\tau)}\left(\psi_{3}^{(1)}\left(\xi^{\prime}, \alpha\right) \psi_{1}^{(1)}\left(\xi, \theta^{-1}(\tau)-\alpha\right)-\right. \\
\left.-\psi_{3}^{(2)}\left(\xi^{\prime}, \alpha\right) \psi_{1}^{(2)}\left(\xi, \theta^{-1}(\tau)-\alpha\right)\right) d \alpha+
\end{gathered}
$$




$$
\begin{gathered}
+\int_{0}^{\theta^{-1}(\tau)}\left(\psi_{3}^{(1)}\left(\xi^{\prime}, \alpha\right) \psi_{2}^{(1)}\left(\xi, \theta^{-1}(\tau)-\alpha\right)-\psi_{3}^{(2)}\left(\xi^{\prime}, \alpha\right) \psi_{2}^{(2)}\left(\xi, \theta^{-1}(\tau)-\alpha\right)\right) d \alpha+ \\
\left.\left.+\left(\psi_{3}^{(1)}\left(\xi^{\prime}, \theta^{-1}(\tau)\right)-\psi_{3}^{(2)}\left(\xi^{\prime}, \theta^{-1}(\tau)\right)\right) \varphi_{\xi_{n} \xi_{n}}(\xi)\right] G\left(x^{\prime}-\xi^{\prime}, \xi_{n}, \theta(t)-\tau\right) d \xi\right)+ \\
+\frac{(\ln a(t))^{\prime}}{\varphi\left(x^{\prime}, 0\right)} \int_{0}^{t}\left(\psi_{3}^{(1)}\left(\xi^{\prime}, \tau\right)-\psi_{3}^{(2)}\left(\xi^{\prime}, \tau\right)\right) f\left(x^{\prime}, t-\tau\right) d \tau- \\
-\left.\frac{1}{\varphi\left(x^{\prime}, 0\right)} \int_{0}^{t}\left(\psi_{3}^{(1)}\left(\xi^{\prime}, \tau\right)-\psi_{3}^{(2)}\left(\xi^{\prime}, \tau\right)\right) f_{t}\left(x^{\prime}, t-\tau\right) d \tau\right|_{T} ^{l} \leqslant \\
\leqslant\left[2\left[\beta_{1}(T) a_{1} a_{0}^{-1} \varphi_{0}^{-1}\left(a_{2}+\varphi_{1}\right)+f_{0} \varphi_{0}^{-1} T_{0}\left(a_{2}+1\right)\right]+8 \beta_{2}(T) a_{1} a_{0}^{-1} \varphi_{0}^{-1}\left(a_{2}+1\right)\left|\psi_{0}\right|_{T_{0}}^{l}\right] \times \\
\times\left|\psi^{(1)}-\psi^{(2)}\right|_{T_{0}}^{l} .
\end{gathered}
$$

Hence, $\left|\left(A \psi^{(1)}-A \psi^{(2)}\right)\right|_{T}^{l}<\rho\left|\psi^{(1)}-\psi^{(2)}\right|_{T_{0}}^{l}$, where $\rho<1$, if $T_{0}$ satisfies the conditions

$$
\begin{gathered}
8 \beta_{0}(T) a_{0}^{-1}\left|\psi_{0}\right|_{T_{0}}^{l} \leqslant \rho<1, \\
2 \beta_{1}(T) a_{0}^{-1}\left(a_{2}+\varphi_{1}\right)+8 \beta_{2}(T) a_{0}^{-1}\left(a_{2}+1\right)\left|\psi_{0}\right|_{T_{0}}^{l} \leqslant \rho<1,
\end{gathered}
$$

$2\left[\beta_{1}(T) a_{1} a_{0}^{-1} \varphi_{0}^{-1}\left(a_{2}+\varphi_{1}\right)+f_{0} \varphi_{0}^{-1} T_{0}\left(a_{2}+1\right)\right]+8 \beta_{2}(T) a_{1} a_{0}^{-1} \varphi_{0}^{-1}\left(a_{2}+1\right)\left|\psi_{0}\right|_{T_{0}}^{l} \leqslant \rho<1$.

It is not difficult to see that from fulfilling the inequalities (4.6) the inequalities (4.5) follows. This indicates that at $T_{0}$ satisfying the conditions (4.6), $A$ satisfies both the properties of a contraction mapping operator for $T<T_{0}$, that is, $A$ realizes contracted mapping of the set $S(T)$ onto itself. Then, according to Lemma 1, in the set $S(T)$ there exists only one fixed point of transformations, that is there exists only one solution to (4.1). Hence, solving the system of (3.1)-(3.3) for example, by the method of successive approximations, we uniquely find the functions $\vartheta(x, t), k\left(x^{\prime}, t\right)$ which belong to $H^{l+2,(l+2) / 2}\left(\mathbb{R}_{T}^{n}\right)$ and $H^{l, l / 2}\left(\mathbb{R}_{T}^{n-1}\right)$, respectively.

Funding. The study of the first author was funded by the Ministry of Innovative Development of the Republic of Uzbekistan, project's no. F-4-02.

\section{REFERENCES}

1. Colombo F. An inverse problem for a parabolic integrodifferential model in the theory of combustion, Physica D: Nonlinear Phenomena, 2007, vol. 236, issue 2, pp. 81-89.

https://doi.org/10.1016/j.physd.2007.07.012

2. Grasselli M. An identification problem for a linear integrodifferential equation occurring in heat flow, Mathematical Methods in the Applied Sciences, 1992, vol. 15, issue 3, pp. 167-186.

https://doi.org/10.1002/mma.1670150304

3. Podio-Guidugli P. A virtual power format for thermomechanics, Continuum Mechanics and Thermodynamics, 2009, vol. 20, no. 8, pp. 479-487.

https://doi.org/10.1007/s00161-009-0093-5

4. Avdonin S., Ivanov S., Wang J. Inverse problems for the heat equation with memory, Inverse Problems and Imaging, 2019, vol. 13, issue 1, pp. 31-38. https://doi.org/10.3934/ipi.2019002

5. Karuppiah K., Kim J.K., Balachandran K. Parameter identification of an integrodifferential equation, Nonlinear Functional Analysis and Applications, 2015, vol. 20, no. 2, pp. 169-185.

https://zbmath.org/?q=an:1321.35259

6. Boumenir A., Tuan V. An inverse problem for the heat equation, Proceedings of the American Mathematical Society, 2010, vol. 138, no. 11, pp. 3911-3921. https://www.jstor.org/stable/25748278

7. Ivanchov M., Vlasov V. Inverse problem for a two-dimensional strongly degenerate heat equation, Electronic Journal of Differential Equations, 2018, vol. 2018, no. 77, pp. 1-17.

https: / / zbmath.org/?q=an:1390.35419 
8. Huntul M.J., Lesnic D., Hussein M.S. Reconstruction of time-dependent coefficients from heat moments, Applied Mathematics and Computation, 2017, vol. 301, pp. 233-253.

https://doi.org/10.1016/j.amc.2016.12.028

9. Hussein M.S., Lesnic D. Simultaneous determination of time and space-dependent coefficients in a parabolic equation, Communications in Nonlinear Science and Numerical Simulation, 2016, vol. 33, pp. 194-217. https://doi.org/10.1016/j.cnsns.2015.09.008

10. Ivanchov M.I., Saldina N.V. Inverse problem for a parabolic equation with strong power degeneration, Ukrainian Mathematical Journal, 2006, vol. 58, no. 11, pp. 1685-1703.

https://doi.org/10.1007/s11253-006-0162-x

11. Hazanee A., Lesnic D., Ismailov M.I., Kerimov N.B. Inverse time-dependent source problems for the heat equation with nonlocal boundary conditions, Applied Mathematics and Computation, 2019, vol. 346, pp. 800-815. https://doi.org/10.1016/j.amc.2018.10.059

12. Hazanee A., Lesnic D., Ismailov M.I., Kerimov N.B. An inverse time-dependent source problems for the heat equation with a non-classical boundary condition, Applied Mathematical Modelling, 2015, vol. 39, issue 20, pp. 6258-6272. https://doi.org/10.1016/j.apm.2015.01.058

13. Hazanee A., Ismailov M.I., Lesnic D., Kerimov N.B. An inverse time-dependent source problem for the heat equation, Applied Numerical Mathematics, 2013, vol. 69, pp. 13-33.

https://doi.org/10.1016/j.apnum.2013.02.004

14. Tanana V.P., Ershova A.A. On the solution of an inverse boundary value problem for composite materials, Vestnik Udmurtskogo Universiteta. Matematika. Mekhanika. Komp'yuternye Nauki, 2018, vol. 28, issue 4, pp. 474-488 (in Russian). https : //doi.org/10.20537/vm180404

15. Wu B., Gao Y., Yan L., Wu S., Wang Z. Existence and uniqueness of an inverse memory kernel for an integro-differential parabolic equation with free boundary, Journal of Dynamical and Control Systems, 2018, vol. 24, pp. 237-252. https://doi.org/10.1007/s10883-017-9372-9

16. Favaron A. Identification of memory kernels depending on time and on an angular variable, $Z$. Anal. Anwend., 2005, vol. 24, issue 4, pp. 735-762. https://doi.org/10.4171/ZAA/1266

17. Durdiev D.K., Rashidov A.Sh. Inverse problem of determining the kernel in an integro-differential equation of parabolic type, Differential Equations, 2014, vol. 50, no. 1, pp. 110-116 (in Russian). https://doi.org/10.1134/S0012266114010145

18. Durdiev D.K., Zhumayev Zh.Zh. Problem of determining a multidimensional thermal memory in a heat conductivity equation, Methods of Functional Analysis and Topology, 2019, vol. 25, no. 3, pp. 219-226. http://mfat.imath. kiev.ua/article/?id=1207

19. Durdiev D.K. On the uniqueness of kernel determination in the integro-differential equation of parabolic type, Journal of Samara State Technical University. Ser. Physical and Mathematical Sciences, 2015, vol. 19, no. 4, pp. 658-666 (in Russian).

https://doi.org/10.14498/vsgtu1444

20. Ladyženskaja O.A., Solonnikov V.A., Ural'ceva N.N. Linear and quasi-linear equations of parabolic type, Providence, RI: American Mathematical Society, 1968.

https://doi.org/10.1090/mmono/023

Original Russian text in Lineinye i kvazilineinye uravneniya parabolicheskogo tipa, Moscow: Nauka, 1967.

21. Romanov V.G. Obratnye zadachi dlya differentsial'nykh uravnenii (Inverse problems for differential equations), Novosibirsk: Novosibirsk State University, 1973.

22. Imanaliev M.I., Asanova K.A., Iskandarov S. The Lyusternik-Sobolev lemma and the specific asymptotic stability of solutions of linear homogeneous Volterra-type integro-differential equations of order 3 , Doklady Mathematics, 2016, vol. 94, pp. 418-422.

https://doi.org/10.1134/S1064562416040190

23. Imanaliev M.I., Iskandarov S. Specific stability criterion for solutions of a fourth-order linear homogeneous Volterra-type integrodifferential equation, Doklady Mathematics, 2009, vol. 79, pp. 231-235. https://doi.org/10.1134/S1064562409020227

24. Kolmogorov A.N., Fomin S.V. Elementy teorii funktsii u funktsional'nogo analiza (Elements of function theory and functional analysis), Moscow: Nauka, 1972. 
Durdiev Durdimurod Kalandarovich, Doctor of Physics and Mathematics, Professor, Department of Mathematics, Bukhara State University, ul. M. Ikbol, 11, Bukhara, 705018, Uzbekistan.

ORCID: https://orcid.org/0000-0002-6054-2827

E-mail: durdiev65@mail.ru

Nuriddinov Javlon Zafarovich, Senior Lecturer, Department of Mathematics, Bukhara State University, ul. M. Ikbol, 11, Bukhara, 705018, Uzbekistan.

ORCID: https://orcid.org/0000-0001-7379-3433

E-mail: j.zafarovich@mail.ru

Citation: D. K. Durdiev, J.Z. Nuriddinov. On investigation of the inverse problem for a parabolic integrodifferential equation with a variable coefficient of thermal conductivity, Vestnik Udmurtskogo Universiteta. Matematika. Mekhanika. Komp'yuternye Nauki, 2020, vol. 30, issue 4, pp. 572-584. 


\section{Д. К. Дурдиев, Ж. 3. Нуриддинов}

\section{Об исследовании обратной задачи для параболического интегро-дифференциального уравне- ния с переменным коэффициентом теплопроводности}

Ключевые слова: интегро-дифференциальное уравнение, обратная задача, ядро, принцип сжимающих отображений.

УДК 517.968.72, 517.958, 536.2

DOI: $10.35634 / \mathrm{vm} 200403$

Исследуется обратная задача определения многомерного ядра интегрального члена, зависящего от временной переменной $t$ и $(n-1)$-мерной пространственной переменной $x^{\prime}=\left(x_{1}, \ldots, x_{n-1}\right)$ из $n$-мерного уравнения теплопроводности с переменным коэффициентом теплопроводности. Прямую задачу представляет задача Коши для этого уравнения. Интегральный член имеет вид свертки по времени ядра и решения прямой задачи. Дополнительное условие для решения обратной задачи задается решение прямой задачи на гиперплоскости $x_{n}=0$. В начале изучаются свойства решения прямой задачи. Для этого эта задача сводится к решению интегрального уравнения второго порядка вольтерровского типа и к нему применяется метод последовательных приближений. Далее поставленная обратная задача приводится к двум вспомогательным задачам, дополнительное условие второй из них содержит неизвестное ядро вне интеграла. Затем вспомогательные задачи заменяются эквивалентной замкнутой системой интегральных уравнений вольтерровского типа относительно неизвестных функций. Применяя метод сжатых отображений к этой системе в классе гёльдеровских функций доказываем основной результат статьи, который является теоремой локального существования и единственности решения обратной задачи.

Финансирование. Исследование первого автора выполнено при финансовой поддержке Министерства Инновационного Развития Республики Узбекистан, проект № F-4-02.

Поступила в редакцию 25.06 .2020

Дурдиев Дурдимурод Каландарович, д. ф.-м. н., профессор, кафедра математики, Бухарский государственный университет, 705018, Узбекистан, г. Бухара, ул. М. Икбол, 11.

ORCID: https: //orcid.org/0000-0002-6054-2827

E-mail: durdiev65@mail.ru

Нуриддинов Жавлон Зафарович, старший преподаватель, кафедра математики, Бухарский государственный университет, 705018, Узбекистан, г. Бухара, ул. М. Икбол, 11.

ORCID: https://orcid.org/0000-0001-7379-3433

E-mail: j.zafarovich@mail.ru

Цитирование: Д.К. Дурдиев, Ж. З. Нуриддинов. Об исследовании обратной задачи для параболического интегро-дифференциального уравнения с переменным коэффициентом теплопроводности // Вестник Удмуртского университета. Математика. Механика. Компьютерные науки. 2020. Т. 30. Вып. 4. С. 572-584. 